https://doi.org/10.22319/rmcp.v9i3.4229

Revisión bibliográfica

\title{
Valor nutricional de la semilla de Mucuna spp. como complemento dietario en animales no rumiantes y rumiantes. Revisión
}

\section{Nutritional value of Mucuna spp. seed, as a dietary complement to non ruminant and ruminant livestock. Review}

\author{
Luis Gerardo Barriada-Bernal ${ }^{\mathrm{a}^{*}}$ \\ Lilia Méndez-Lagunas ${ }^{b}$ \\ Juan Rodríguez-Ramírez ${ }^{\mathrm{b}}$ \\ Sadoth Sandoval-Torres ${ }^{\mathrm{b}}$ \\ Laura Aquino-González ${ }^{\mathrm{b}}$
}

${ }^{a}$ Consejo Nacional de Ciencia y Tecnología. Centro Interdisciplinario de Investigación para el Desarrollo Integral Regional, Unidad Oaxaca. Hornos 1003, Colonia Noche Buena; 71230, Santa Cruz Xoxocotlán, Oaxaca, México. Teléfono 01(55)9515170610 ext. 82753.

${ }^{\mathrm{b}}$ Instituto Politécnico Nacional. Centro Interdisciplinario de Investigación para el Desarrollo Integral Regional, Unidad Oaxaca. México.

*Autor de correspondencia: lgbarriada@ conacyt.mx

\section{- Resumen:}

Dentro de la industria pecuaria, el diseño de dietas adecuadas para la productividad y salud de los animales es esencial. Para lo anterior, comúnmente se emplean raciones que combinan alimentos de volumen (aporte de fibra) y alimentos concentrados como los cereales (aporte energético), leguminosas (aporte proteico) y concentrados minerales. Mucuna spp., es una leguminosa tropical cuyas características nutricionales, para el área pecuaria, han sido subaprovechadas. La semilla de mucuna posee características nutricias de importancia para el sector pecuario tales como: 1) concentración (base seca) de proteína cruda y energía de 20 
al $30 \%$ y de 15.7 a $24.7 \mathrm{MJ} \mathrm{kg}^{-1}$, respectivamente, 2) presencia de ácidos grasos insaturados, y 3) concentración de anti-nutrientes similares a otras leguminosas dentro de los cuales la concentración de la L-3,4 dihidroxifenilalanina (L-DOPA,) puede ser fácilmente reducida hasta no provocar efectos adversos en el crecimiento y desarrollo animal. Adicionalmente, la semilla de mucuna es un alimento económicamente ventajoso, ya que se considera como residuo de otras prácticas agrícolas. El propósito de este trabajo es presentar una revisión de la información disponible respecto a la concentración de componentes nutricionales presentes en la semilla de Mucuna spp. de interés para sistemas de producción y crianza animal.

- Palabras clave: Proteínas, Aminoácidos, Carbohidratos, Ácidos grasos, Fibra cruda, Antinutrientes, Mucuna.

\section{- Abstract:}

Adequate design of diets to animal health and productivity is essential in livestock industry. For the above, commonly are use bulky ingredients (fibrous sources) combined with a concentrates ingredients as cereals (energy sources), legumes (protein sources) and mineral concentrates. Mucuna spp. is a tropical legume in which its nutrients characteristics have been underutilized by livestock industry. Mucuna seed have an important nutritional characteristics as feedstuff: 1) Crude protein and energy concentration (dry matter basis) of 20 to $30 \%$ and 15.7 to $24.7 \mathrm{MJ} \mathrm{kg}^{-1}$, respectively; 2) Presence of unsaturated fatty acids; 3 ) Similar concentration of anti-nutrient factors than other legumes, within which L-3,4 dihidroxiphenilalanine (L-DOPA,) can be easy reduced until no adverse effects on animal growth are noted. In addition, mucuna seed is a feedstuff economically advantageous, since is commonly considered as agriculture waste. The aim of this paper is to present a review of available information about the presence and concentration of nutritional compounds in the seed of Mucuna spp. with high potential for livestock industry.

- Key words: Protein, Aminoacids, Carbohydrates, Unsaturated acids, Crude fiber, Antinutrients, Mucuna.

Recibido 16/07/2016

Aceptado 01/11/2017 


\section{| Introducción \|}

Actualmente, la industria pecuaria experimenta una fuerte presión, derivada de múltiples factores que la limitan para poder satisfacer la demanda creciente por parte de los mercados nacionales, de proteína de origen animal con características nutricionales específicas ${ }^{(1)}$.

La industria pecuaria emplea una limitada variedad de fuentes de alimento, tanto de origen vegetal como animal, especialmente aquéllas que también son empleadas como ingrediente principal en productos procesados destinados a la nutrición humana; por lo que este factor limita significativamente su desarrollo. El empleo de alimentos derivados de fuentes que son sub aprovechadas, y que poseen características nutricias tales que puedan ser empleadas en las dietas de los animales de cría/engorda, se constituyen en una opción que puede mejorar la competitividad de la industria ${ }^{(2,3,4)}$.

La formulación de dietas para la cría/engorda de rumiantes y no rumiantes, busca cubrir requerimientos específicos, tales, que permitan mejorar la capacidad de ingestión, mejorar la deposición proteica sobre los tejidos del animal en función del peso, así como el equilibrio entre los costos de los alimentos y las ganancias derivadas del aprovechamiento del animal ${ }^{(5)}$.

En las zonas tropicales y subtropicales existe una amplia variedad de recursos que son factibles de ser utilizados en la alimentación animal. Entre estas opciones se incluyen los granos de especies consideradas como no aprovechadas o como cosechas menores. Мисипа spp. es una herbácea anual de características taxonómicas y nutricias similares a otras leguminosas (Phaseolus vulgaris, Glycine max, entre otras), pero con un alto grado de variabilidad en sus propiedades nutricionales intra e interespecíficas, propias de especies con un grado bajo de domesticación, y cultivada bajo una amplia variedad de condiciones edafoclimáticas ${ }^{(6,7)}$.

La clase de semillas a la que pertenece mucuna, se le denomina como "de interés", y comprenden a aquellas fuentes alimenticias no tradicionales, que tienen la presencia y concentración de compuestos de alto valor económico/nutricional (referidos principalmente a aminoácidos y carbohidratos), con potencial de convertirse en un producto agropecuario de alto valor ${ }^{(8)}$. La semilla de mucuna presenta compuestos reconocidos como antinutricionales y cuya sola presencia en las raciones dietarias podría reducir la disponibilidad biológica y la digestibilidad de uno o más nutrientes, ocasionando la disminución de la función digestiva, la eficiencia reproductiva y la respuesta inmune ${ }^{(9,10)}$. Existen experiencias exitosas que indican que las concentraciones de dichos compuestos antinutricionales, no son un 
impedimento en su empleo en las dietas para animales rumiantes ${ }^{(11,12)}$ y no rumiantes ${ }^{(13,14,15)}$ destinados al consumo humano.

En la presente revisión, se analizan los diferentes compuestos en Mucuna spp. discutiendo su posible valor nutricio como alimento alternativo o como complemento dietario en animales de crianza para consumo humano.

\section{- Aporte energético}

Cubrir las necesidades energéticas mediante el empleo adecuado de ingredientes en las dietas para animales, es un apartado de gran importancia dentro del área pecuaria ${ }^{(16)}$, debido a que los costos de energía para el mantenimiento de los animales representa del 60 al $80 \%$ del total de la energía consumida en el sistema de crianza ${ }^{(16,17)}$. El empleo de granos y pastos, con o sin adición de complementos, así como aportes dietarios derivados de la ingesta no controlada de otras especies vegetales, constituyen la fuente principal de energía para animales de crianza $^{(18)}$.

La mayoría de los carbohidratos de la semilla de mucuna son azúcares no reductores; siendo los almidones los de mayor concentración (Cuadro 1). De acuerdo al contenido de almidones presentes en diferentes especies de mucuna se infieren variaciones intra e interespecificas importantes; entre las especies reportadas, Mucuna preta es la de mayor concentración, 41.17 $\%^{(19)}$, mientras que $M$. veracruz var. cáscara moteada es la de menor concentración, 22.9 $\%^{(20)}$. En cuanto a los azúcares reductores, estos representan apenas entre el 3 al $5 \%$ de la semilla, siendo $M$. deeringeana la de mayor concentración, $5.5 \%{ }^{(20)}$, y $M$. ghana la de menor concentración $1.5 \%{ }^{(19)}$. 
Cuadro 1: Carbohidratos presentes en semillas de mucuna

\begin{tabular}{|c|c|c|c|c|c|}
\hline & 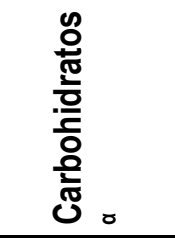 & 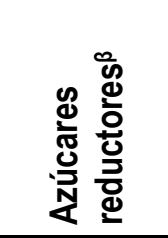 & 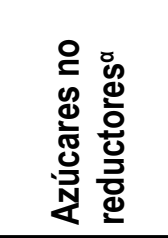 & $\begin{array}{l}\text { 듬 } \\
\text { 음을 } \\
\text { 든 }\end{array}$ & 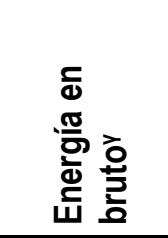 \\
\hline M. pruriens $(20)$ & $44.9 \pm 0.25$ & $334 \pm 3.61$ & $2.56 \pm 0.34$ & $37.5 \pm 0.31$ & $22.7 \pm 0.23$ \\
\hline M. pruriens ${ }^{(36)}$ & 62.43 & 237 & 1 & 40.5 & 17.17 \\
\hline M. cochinchinesis ${ }^{(20)}$ & $44.8 \pm 0.35$ & $430 \pm 4.04$ & $3.78 \pm 0.45$ & $36.8 \pm 0.23$ & $21.8 \pm 0.45$ \\
\hline M. rajada $(20)$ & $49.7 \pm 0.23$ & $554 \pm 4.85$ & $4.01 \pm 0.48$ & $40.1 \pm 0.34$ & $22.9 \pm 0.35$ \\
\hline M. veracruz (cáscara blanca)(20) & $46.4 \pm 0.34$ & $480 \pm 4.56$ & $2.66 \pm 0.06$ & $39 \pm 0.29$ & $23.1 \pm 0.39$ \\
\hline M. veracruz (cáscara moteada) ${ }^{(20)}$ & $45.6 \pm 0.18$ & $301 \pm 3.60$ & $4.14 \pm 0.1$ & $22.9 \pm 0.22$ & $22.9 \pm 0.29$ \\
\hline M. deeringeana(20) & $45.2 \pm 0.2$ & $550 \pm 3.20$ & $3.3 \pm 0.27$ & $24.7 \pm 0.26$ & $24.7 \pm 0.12$ \\
\hline M. utilis (cáscara blanca) (36) & l & l & l & l & 16 \\
\hline M. utilis (cáscara negra) (35) & I & 1 & I & l & 16.4 \\
\hline M. utilis $s^{(35)}$ & 59.27 & 154 & l & 39.43 & 16.9 \\
\hline M. monoesperma ${ }^{(36)}$ & 1 & 1 & I & 1 & 18 \\
\hline M. gigantea(35) & 1 & l & l & 1 & 15.7 \\
\hline M. pruriens (begur) $)^{(56)}$ & 1 & 1 & 1 & 1 & $16.5 \pm 0.57$ \\
\hline M. pruriens var IRZ(56) & I & 1 & 1 & 1 & $17.2 \pm 0.33$ \\
\hline M. georgia(36) & 61.3 & 283 & 1 & 40.89 & 16.83 \\
\hline M. ghana(36) & 62.78 & 150 & l & 40.13 & 16.67 \\
\hline M. $\operatorname{preta}^{(36)}$ & 66.47 & 300 & 1 & 41.17 & 16.7 \\
\hline M. jaspeada(36) & 63.74 & 319 & 1 & 39.22 & 16.66 \\
\hline Phaseolus vulgaris ${ }^{(23,24)}$ & 1 & 1 & $2.5-5$ & $34-48$ & 13.9 \\
\hline$\underline{\text { Helianthus annuus }}^{(22)}$ & l & 1 & 2.2 & 0.5 & 25.3 \\
\hline Gossypium spp. (22) & l & l & 0.9 & 0 & 21.3 \\
\hline Zea mays(21) & 1 & 1 & 1.7 & 72 & 14.8 \\
\hline
\end{tabular}

a Valores porcentuales promedio en la semilla en base seca.

$\beta \mathrm{mg} 100 \mathrm{~g}^{-1}$ de semilla en base seca.

Y MJ kg-1 en la semilla en base seca.

El contenido de almidón de la semilla de mucuna representa entre el 16 y $41 \%$ del contenido de almidón del grano de Zea mays, maíz ${ }^{(21)}$; que está por encima de otras semillas forrajeras de interés como Helianthus annuus, girasol; con concentraciones porcentuales promedio de $0.5 \%{ }^{(22)}$. En comparación con otras leguminosas como Phaseolus vulgaris (frijol común), las semillas de mucuna tienen concentraciones comparables de almidón ${ }^{(23,24)}$.

El aporte energético de los carbohidratos dentro de las dietas para animales, está condicionado, por completo, por el tipo de aparato digestivo del animal, rumiante o no rumiante. La eficiencia parcial del aporte energético obtenido a través de los procesos 
fermentativos, digestivos y metabólicos, está estrechamente relacionada con el aporte de energía neta para el mantenimiento y producción ${ }^{(25)}$.

La capacidad de empleo de la energía proveniente de los carbohidratos presentes en la dieta de los animales difiere entre especies rumiantes y no rumiantes ${ }^{(26)}$. En rumiantes, del 60 al $80 \%$ de los carbohidratos ingeridos son fermentados en el rumen produciendo ácidos grasos volátiles (AGV) y energía para la síntesis microbiana. Los AGV son absorbidos a través de la pared rumial y empleados como energía por el animal. De los carbohidratos que escapan a la fermentación ruminal, una parte es digerida y absorbida en el tracto posterior ${ }^{(27,28)}$.

El aporte energético y su empleo en sistemas pecuarios de semillas de mucuna son poco conocidos. En dietas para rumiantes se han reportado $9.7 \mathrm{MJ} \mathrm{kg}^{-1}$, mientras que reportan valores de EM de $15.48 \mathrm{MJ} \mathrm{Kg}^{-1}$ en dietas suplementadas para no rumiantes ${ }^{(13,14)}$. Estos valores se encuentran debajo de los reportados por la Fundación Española para el Desarrollo de la Nutrición Animal ${ }^{(22)}$ en una amplia variedad de semillas (Cuadro 2). Los valores reportados sugieren que la digestibilidad de mucuna es baja, indicando la presencia e interacción de compuestos antinutricionales (polifenoles, inhibidores de la tripsina, altas cantidades de fibra, entre otros); de dietas constituidas de manera inadecuada, así como de combinaciones, en cantidad y variedad, de ingredientes incorrectos ${ }^{(29)}$.

Cuadro 2: Digestibilidad y energía metabolizable de leguminosas

\begin{tabular}{|c|c|c|c|c|}
\hline & $\begin{array}{c}\text { Energía } \\
\text { metabolizable }^{\alpha} \\
\text { (Bovinos) }\end{array}$ & Digestibilidad & $\begin{array}{c}\text { Energía } \\
\text { metabolizable } \\
\text { (Porcinos) }\end{array}$ & Digestibilidad \\
\hline Mucuna prurensis ${ }^{(13,14)}$ & 9.7 & $0.63-0.69$ & 15.48 & 0.55 \\
\hline Glycine $\max ^{(22)}$ & 14.95 & 0.9 & 16.90 & 0.84 \\
\hline$\underline{\text { Helianthus annuus }}^{(22)}$ & 18.60. & 0.86 & 18.8 & 0.80 \\
\hline$\overline{\text { Gossypium spp(22) }}$ & 12.6 & 0.8 & 13.3 & 0.55 \\
\hline Zea mays ${ }^{(22)}$ & 11.71 & 0.65 & 14.22 & 0.73 \\
\hline
\end{tabular}

a MJ Kg-1 en la semilla en base seca.

El aumento en la digestibilidad de mucuna puede abordarse a partir de varios factores: i) la reducción de los compuestos antinutricionales, ya que múltiples referencias indican que el empleo de una extracción acuosa a temperaturas moderadas $\left(40\right.$ a $\left.50{ }^{\circ} \mathrm{C}\right)$ reduce significativamente la concentración de compuestos fenólicos (fenoles, taninos y saponinas) y farmacológicos (L-3,4 dihidroxifenilalanina, L-DOPA), así como la inhibición de la actividad biológica de los compuestos enzimáticos (inhibidores de la tripsina), sin afectar significativamente la mayoría de las características nutricias de interés ${ }^{(30-33)}$; ii) la reducción del contenido de fibra cruda en las dietas ${ }^{(34)}$. La fibra cruda oscila entre $11.55 \%$ del peso 
seco de la semilla de Mucuna utilis var. cáscara blanca ${ }^{(35)}$, y el $1.54 \%$ del peso seco de la semilla de M. utilis $^{(36)}$ colocándola entre el rango para Zea mays y Glycine max, 5 y $16 \%{ }^{(37)}$, pero superior a Phaseolus vulgaris, 1.77 a $2.77 \%{ }^{(37)}$.

\section{- Ácidos grasos •}

El empleo de ácidos grasos en la formulación de las dietas para rumiantes y no rumiantes, se lleva a cabo cuando se desea aumentar o complementar la ingesta calórica; sobre todo en aquellas situaciones en que el apetito se ve restringido, como escasez, mala formulación de la dieta, o cuestiones sanitarias ${ }^{(38,39)}$.

Así mismo, se ha correlacionado el efecto del aumento en la ingesta de ácidos insaturados con una reducción de la concentración de grasas saturadas sobre los tejidos musculares del animal. Diferentes cereales se suministran al ganado en su forma entera, adicionados directamente a la ración, o bien molidos o granulados, debido a la presencia mayoritaria de ácidos insaturados de cadena corta ${ }^{(40)}$. Comúnmente, las semillas empleadas para suplementar con ácidos grasos las dietas animales, son semillas oleaginosas como Gossypium spp, Helianthus annuus y Carthamus tinctorius; y en menor grado Glicine max, Vicia faba y Linum usitatissimum ${ }^{(5)}$.

En semillas de la especie Mucuna spp., existen pocas referencias del perfil y concentración de ácidos grasos ${ }^{(35,36)}$. Se han reportado 11 ácidos grasos identificados, siendo los ácidos poliinsaturados los de concentración relativa más baja (ácido oléico 14 a $39 \%$, ácido linoleico 21 a $44 \%$, y ácido linolénico 21 a $44 \%$ ). Los perfiles de ácidos grasos muestran diferencias intra e interespecíficas ${ }^{(35,36)}$, particularmente en la presencia/ausencia de ácidos del complejo omega 9 ( $\left.\mathrm{C}_{16 \mathrm{n}-\mathrm{n}-9}, \mathrm{C}_{20: \mathrm{n}-9}, \mathrm{C}_{22: \mathrm{n}-9}\right)$, de ácido behénico $\left(\mathrm{C}_{20: 0}\right)$, y de ácido lingocérico $\left(\mathrm{C}_{24: 0}\right)$. La concentración porcentual de ácidos grasos saturados e insaturados en la semilla de mucuna la hace ideal para incluirla en complementos dietarios.

No existe información relacionada con la evaluación sensorial, metabólica o de aspectos relacionados con la engorda de animales, evaluados desde el punto de vista de raciones dietéticas enriquecidas con ácidos grasos provenientes de semillas de mucuna, ya sea en forma de la adición de semillas enteras, harina, o como un complemento adicionado a los alimentos balanceados para animales. 


\section{Contenido proteico}

Una evaluación del aporte proteínico de semillas de Mисиna spp. en dietas animales índica un contenido entre el 20 al $30 \%$ (Cuadro 3); siendo la fracción globular la de mayor concentración. Al igual que otras semillas, la fracción y la concentración de proteína parecen ser afectadas por el genotipo y condiciones edafoclimáticas ${ }^{(41,42)}$. Mohan y Janardhanan ${ }^{(35)}$ realizaron un análisis intra e inter específico (Mucuna utilis variedades cáscara blanca y negra; y $M$. monosperma) determinando valores de 27 a $29 \%$ de proteína cruda total; reportando a las globulinas como las de mayor concentración (11 a $14 \%$ del contenido proteínico total), y las prolamidas las de menor presencia $(0.68 \%$ a 0.89$)$.

Cuadro 3: Proteínas en semillas de Mucuna spp.

\begin{tabular}{|c|c|c|c|c|c|c|c|}
\hline & 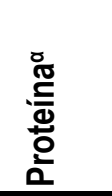 & 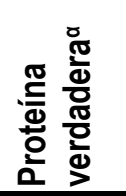 & 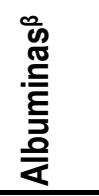 & 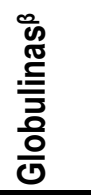 & 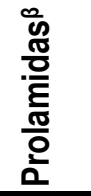 & 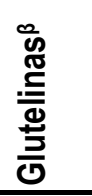 & $\begin{array}{l}\bar{\pi} \\
\text { 응 }\end{array}$ \\
\hline M. cochinchinesis $^{(20)}$ & 38.4 & 32.3 & l & l & l & l & 70.7 \\
\hline M. rajada(20) & 33.2 & l & l & l & l & l & 33.2 \\
\hline M. veracruz (cáscara blanca)(20) & 37.8 & 31.8 & l & l & l & l & 69.6 \\
\hline M. deeringeana(20) & 38.2 & 1 & 1 & 1 & 1 & 1 & 38.2 \\
\hline M. utilis (cáscara blanca)(35) & 25.7 & 23.4 & 8.39 & 11.7 & 0.76 & 1.92 & 71.87 \\
\hline M. utilis (cáscara negra)(35) & 29.6 & 22.73 & 8.32 & 12.5 & 0.89 & 1.75 & 75.79 \\
\hline M. utilis ${ }^{(36)}$ & 29.62 & I & 1 & 1 & 1 & 1 & 29.62 \\
\hline M. monoesperma ${ }^{(35)}$ & 23.5 & 20.4 & 4.22 & 14.0 & 3.34 & 8.45 & 73.91 \\
\hline M. pruriens ${ }^{(20)}$ & 37.5 & 31.6 & I & 1 & 1 & 1 & 69.1 \\
\hline M. pruriens ${ }^{(13)}$ & 28.9 & 17.91 & 1 & l & 1 & l & 46.81 \\
\hline M. pruriens var IRZ(36) & 27.17 & 1 & I & l & I & l & 27.17 \\
\hline M. georgia(36) & 29.31 & l & 1 & 1 & 1 & 1 & 29.31 \\
\hline M. ghana(36) & 29.16 & l & l & l & l & l & 29.16 \\
\hline M. preta(35) & 27.95 & l & l & l & l & l & 27.95 \\
\hline M. jaspeada(35) & 27.56 & l & I & l & l & I & 27.56 \\
\hline
\end{tabular}

a Valores promedio porcentuales en la semilla en base seca.

$\beta$ Valores expresados en gramos de la fracción proteica $100 \mathrm{~g}^{-1}$ de semilla en base seca.

I= no presente, no cuantificado, o por debajo del límite de cuantificación. 
Adebowale $e t a l^{(20)}$ realizaron así mismo un análisis intra e interespecífico (Mucuna pruriens, M. cochinchinensis, M. rajada, M. deeringeana, M. veracruz blanca y M. veracruz moteada); reportando contenidos promedio de $27 \%$ de proteína cruda total. Resultados similares se han obtenido al caracterizar las proteínas crudas totales en otras poblaciones de $M$. prurensis, en donde el contenido reportado, en base seca, se sitúa entre 27.9 y $30.1 \%{ }^{(31,43)}$.

Al comparar los valores reportados para otras especies forrajeras, la semilla de mucuna exhibe contenidos de proteína cruda mayores a los considerados para las leguminosas, 20 a $26 \%{ }^{(21)}$, encontrándose dentro de los rangos de las semillas consideradas como altas en contenidos de proteína, a Gossypium spp., con $31 \%{ }^{(37)}$. En cuanto a la digestibilidad, se han realizado ensayos in $v i v o^{(13)}$ con coeficientes $\approx 50 \%$ en no rumiantes; mientras que en ensayos in vitro ${ }^{(35)}$, los coeficientes se encuentran en un rango de 64 a $71 \%$. Estos valores son ligeramente inferiores para los reportados en pruebas in vivo ${ }^{(22)}$ para semillas de Helianthus annuиs y Zea mays en alimentación de rumiantes, cerdos y aves (86, 80 y $89 \%$ para semillas de $H$. annuиs, y de 65,73 y $83 \%$ para semillas de Z. mays). Se ha reportado la digestibilidad de proteínas de mucuna por encima del rango de otras semillas de uso intensivo en el área pecuaria, como Glicine max y Oriza sativa y de otras leguminosas como Phaseolus vulgaris $^{(44)}$.

Experiencias con animales, principalmente no rumiantes, mostraron que no existen diferencias significativas (en los pesos finales de los animales, así como en la retención y disponibilidad de nitrógeno) cuando se suplementó como parte de las dietas con semilla pre tratada de mucuna ${ }^{(45,46,47)}$.

\section{- Aminoácidos}

Existe escasa información con respecto al perfil de aminoácidos obtenidos a partir de los extractos proteínicos de las semillas de Mucuna spp. Adebowale et al ${ }^{(20)}$ reportaron 18 aminoácidos, siendo la prolina, el ácido aspártico, la glutamina y el ácido glutámico, los de mayor concentración. Los aminoácidos esenciales para la nutrición pecuaria se muestran en el Cuadro 4. 
Cuadro 4: Aminoácidos identificados en semillas de Mucuna spp.

\begin{tabular}{|c|c|c|c|c|c|c|c|c|c|c|}
\hline & 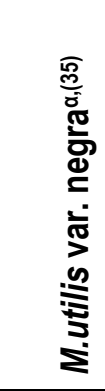 & 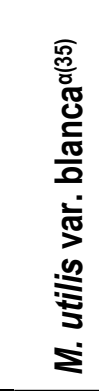 & 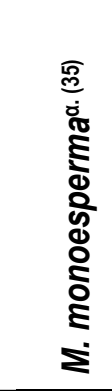 & 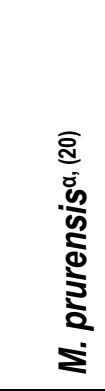 & 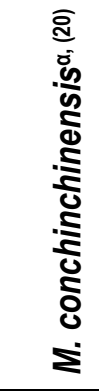 & 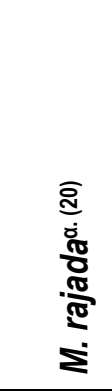 & 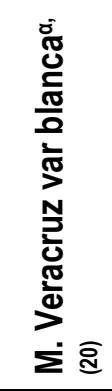 & 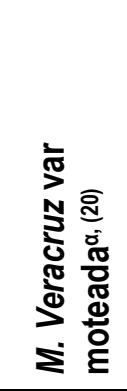 & 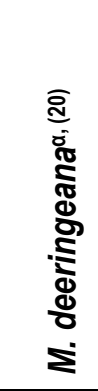 & 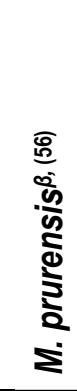 \\
\hline Histidina & 29.3 & 30.1 & 26.8 & 36.7 & 23.6 & 35.7 & 37.9 & 45.9 & 44.9 & 3.47 \\
\hline Isoleucina & 72.0 & 80.2 & 63.1 & 96.7 & 90.8 & 88.7 & 89.5 & 88.6 & 98.5 & 4.16 \\
\hline Leucina & 66.8 & 66.1 & 51.9 & 77.8 & 72.7 & 67.8 & 70.7 & 75.8 & 80.4 & 7.88 \\
\hline Lisina & 56.1 & 59.8 & 52.4 & 78.5 & 67.8 & 56.7 & 80.4 & 74.5 & 79.5 & 6.18 \\
\hline Metionina cisteína & 27.0 & 1 & 1 & 1 & 23.6 & 12.6 & 1 & 1 & 22.0 & 1.07 \\
\hline Fenilalanina tirosina & 89.3 & 184 & 93.7 & 91.8 & 100 & 109.9 & 100.2 & 78 & 71.7 & 9.35 \\
\hline Treonina & 49.1 & 12.7 & 33.6 & 46.7 & 50.4 & 54.4 & 66.3 & 97.4 & 60.8 & 3.58 \\
\hline Triptófano & 1 & 1 & 1 & 20.9 & 23.4 & 34.3 & 23.4 & 21.3 & 21.2 & 1.22 \\
\hline Valina & 33.9 & 45.9 & 44.9 & 57.8 & 69.8 & 69.4 & 66.8 & 62.2 & 76.4 & 4.23 \\
\hline Proteína total & 75.7 & 71.8 & 73.9 & 69.1 & 70.7 & 33.2 & 69.6 & 37.9 & 38.2 & 69.1 \\
\hline
\end{tabular}

/ No presente, no cuantificado, o por debajo del límite de cuantificación.

a gramos de aminoácido $\mathrm{kg}^{-1}$ de semilla en base seca.

$\beta$ valores calculados en gramos de aminoácido $\mathrm{g}^{-1}$ de nitrógeno total cuantificado.

Diferencias intra e interespecíficas se acentúan en algunos de los aminoácidos; por ejemplo, la combinación metionina/cisteína y el triptófano; cuyas concentraciones determinadas en más de la mitad de las especies y variedades de mucuna descritas, se encuentran en concentraciones tales que se les considera como los aminoácidos limitantes; mientras que en otras especies se encuentran por arriba de los valores de otras leguminosas (p. ej. Glicine max). Este comportamiento se observa en semillas de otras especies, sobre todo en aquéllas que no han sido sujetas a procesos de manejo/mejoramiento agrícola ("domesticación"). Independientemente de la especie o variedad evaluada, las semillas de mucuna son una fuente importante de leucina y lisina. 


\section{- Minerales •}

En las semillas de mucuna, se ha documentado la presencia de siete de los nueve macrominerales esenciales, minerales de requerimientos de más de $70 \mathrm{mg} \mathrm{kg}^{-1}$ de peso del animal (Cuadro 5); siendo deficientes en compuestos azufrados y clorados. Las deficiencias se acentúan dentro del grupo de los microminerales (requerimientos menores de $70 \mathrm{mg} \mathrm{kg}^{-1}$ de peso), donde sólo están presentes 4 de los 14 considerados como esenciales ${ }^{(21)}$.

Cuadro 5: Minerales presentes en semillas de mucuna

\begin{tabular}{lccccccccc}
\hline & $\mathrm{Na}^{\alpha}$ & $\mathrm{K}^{\alpha}$ & $\mathrm{Ca}^{\alpha}$ & $\mathbf{M g}^{\alpha}$ & $\mathbf{P}^{\alpha}$ & $\mathrm{Fe}^{\alpha}$ & $\mathrm{Cu}^{\alpha}$ & $\mathbf{Z n}^{\alpha}$ & $\mathbf{M n}^{\alpha}$ \\
\hline M. cochinchinesis(20) & 0.002 & 0.004 & 0.293 & 0.05 & 0.568 & 0.015 & 0.001 & 0.002 & 0 \\
M. rajada(20) & 0.002 & 0.41 & 0.258 & 0.06 & 0.601 & 0.009 & 0.001 & 0.002 & 0 \\
M. veracruz (cáscara blanca)(20) & 0.004 & 0.433 & 0.359 & 0.07 & 0.459 & 0.014 & 0 & 0.004 & 0 \\
M. veracruz (cáscara moteada)(20) & 0.003 & 0.356 & 0.008 & 0.059 & 0.566 & 0.02 & 0 & 0.004 & 0 \\
M. deeringeana(20) & 0.004 & 0.433 & 0.359 & 0.07 & 0.459 & 0.014 & 0 & 0.004 & 0 \\
M. utilis (cáscara blanca)(35) & 0.071 & 1.66 & 0.759 & 0.271 & 0.327 & 0.006 & 0 & 0.002 & 0.009 \\
M. utilis (cáscara negra)(35) & 0.004 & 0.361 & 0.408 & 0.073 & 0.607 & 0.018 & 0.001 & 0.006 & 0 \\
M. gigantean(56) & $/$ & 0.23 & 0.518 & $/$ & 0.194 & $/$ & $/$ & $/$ & $/$ \\
M. utilis(35) & $/$ & 0.003 & 0.0002 & 0.0002 & 0.0005 & 0.01 & 0.004 & 0.005 & 0.006 \\
M. monoesperma(35) & 0.062 & 1.761 & 0.172 & 0.072 & 0.129 & 0.005 & 0 & 0.001 & 0.008 \\
M. pruriens(20) & 0.003 & 0.389 & 0.262 & 0.053 & 0.457 & 0.015 & 0.001 & 0.004 & 0 \\
M. pruriens(56) & $/$ & 1.11 & 0.25 & $/$ & 0.22 & $/$ & $/$ & $/$ & $/$ \\
M. pruriens, var IRZ(36) & $/$ & 0.005 & 0.0001 & 0.0002 & 0.0005 & 0.010 & 0.001 & 0.003 & 0.006 \\
M. georgia(36) & $/$ & 0.003 & 0.0001 & 0.0002 & 0.0005 & 0.009 & 0.002 & 0.003 & 0.007 \\
M. ghana(36) & $/$ & 0.005 & 0.0001 & 0.0002 & 0.0004 & 0.010 & 0.003 & 0.003 & 0.006 \\
M. preta(36) & $/$ & 0.005 & 0.0001 & 0.0002 & 0.0005 & 0.009 & 0.002 & 0.004 & 0.006 \\
M. jaspeada(36) & $/$ & 0.008 & 0.0001 & 0.0002 & 0.0005 & 0.006 & 0.001 & 0.004 & 0.005 \\
\hline
\end{tabular}

a Los valores se expresan en gramos $100 \mathrm{~g}^{-1}$ de semilla en base seca.

I No presente, no cuantificado, o por debajo del límite de cuantificación.

Además de los nutrientes mencionados, existen diferencias intraespecíficas e interespecíficas aparentemente condicionadas por variables edafoclimáticas y estado fenológico del grano. Mucuna utilis var. cáscara blanca ${ }^{(35)}$, es la de mayor concentración de macrominerales, particularmente de aquéllos de mayor interés por su alto costo, los cuales son suplementados en las dietas (sodio, $0.071 \mathrm{~g}$; calcio, $0.408 \mathrm{~g}$; fósforo, $0.327 \mathrm{~g}$ por $100 \mathrm{~g}^{-1}$ de semilla). Por otra

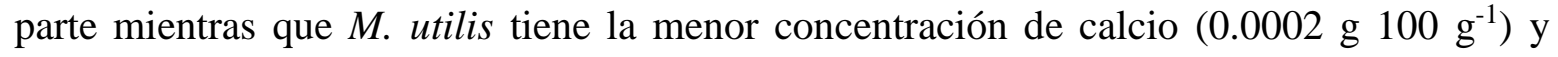
fósforo $\left(0.0005 \mathrm{~g}_{\text {por }} 100 \mathrm{~g}^{-1}\right)^{(35)}$.

En comparación con otras semillas, las concentraciones de macrominerales y microminerales son claramente inferiores a la mayoría de los cereales de uso intensivo, lo que constituye un 
claro indicativo de la necesidad de suplementar las dietas animales con sales minerales cuando el principal ingrediente sean las semillas de mucuna. Al incorporar la semilla de mucuna en dietas animales, se debe elegir preferentemente las proporciones que mejoren la relación disponibilidad / costos de los complementos minerales.

\section{- Antinutrientes}

Los factores antinutrientes son compuestos, que al ser ingeridos, son adversos desde el punto de vista nutricional, ya que reducen el consumo de alimentos, modifican el proceso digestivo normal, así como la absorción y la utilización de nutrientes ${ }^{(48)}$.

Cuando se habla de material vegetal, a los metabolitos secundarios se les considera como los antinutrientes ${ }^{(49,50)}$. Son mecanismos de defensa inducidos y contra constitutivos contra la herbívora de animales e insectos, la infección de microorganismos, y como respuesta sistémica a condiciones medioambientales estresantes ${ }^{(49,50)}$.

Los componentes antinutricionales más importantes en semillas de Mucuna spp. son: i) la LDOPA, siendo el anti nutriente que puede catalogarse como "limitante" en el aprovechamiento de mucuna, ii) poli fenoles, iii) taninos, iv) inhibidores de proteasas (siendo los de mayor concentración), v) lecitinas. El contenido de fenoles totales varía de 30 a 90 g $\mathrm{kg}^{-1}$ de semilla ${ }^{(20,35)}$. La L-DOPA se mantiene entre el 3 al $7 \%{ }^{(35)}$, siendo M. deeringeana la de menor concentración, $3.87 \%{ }^{(35)}$.

A los compuestos considerados como antinutrientes se les atribuyen efectos adversos como la diminución de la capacidad de digestión de los carbohidratos, proteínas, disminuyendo la capacidad enzimática (p. ej. inhibiendo a la amilasa, la tripsina, la cromotripisina, la lipasa, entre otras); la absorción de vitaminas y minerales; causado por el daño sobre la mucosa del tracto digestivo ${ }^{(48)}$.

La presencia y concentración de L-DOPA ha sido objeto de debate, ya que algunas investigaciones sugieren que tiene un efecto negativo en el desarrollo normal del animal ${ }^{(51,52)}$, mientras que otras concluyen que ésta no afecta significativamente ${ }^{(53,54)}$.

Aunque se reconocen los efectos fitohematoglutinantes de las fracciones proteicas de mucuna en humanos ${ }^{(35)}$, no existen estudios de sus efectos en animales rumiantes y no rumiantes; pero podría suponerse efectos adversos en la nutrición de los animales ${ }^{(55)}$. 
En comparación con las semillas de Mисuna spp., las semillas forrajeras presentan una gama más amplia de compuestos antinutricionales ${ }^{(37)}$, siendo la presencia de inhibidores enzimáticos los de mayor espectro y diversidad. La presencia de saponinas, polifenoles, fitohemaglutininas e inhibidores enzimáticos, parece ser común a las especies forrajeras y a mucuna.

\section{\| Conclusiones \|}

El alto contenido de proteínas, almidones, ácidos grasos presentes en las semillas de mucuna puede ser empleado como complemento en la formulación de las dietas para animales rumiantes y no rumiantes.

Debido al alto contenido de proteínas, una de las aplicaciones más interesantes de la semilla radica en su empleo como suplemento dietario mediante su dosificación directa (semilla entera, o molida) y como productos proteínicos procesados (concentrados o aislados proteínicos).

Si bien existe evidencia del efecto de los compuestos antinutricionales, principalmente de la acción biológica de la L-DOPA, sobre el estado nutricional de los animales de cría/engorda; existen alternativas tecnológicas simples, eficaces y de bajo costo que reducen la concentración de antinutrientes en las semillas de mucuna, hasta niveles mínimos, en donde no se registra diferencia significativa con respecto a las dietas tradicionales.

La siguiente fase de evaluación del empleo de mucuna como alimento alternativo para la industria pecuaria, consiste en: i) el desarrollo de productos alimenticios con mejores índices de digestibilidad proteica, energía metabolizable y contenido de fibra; ii) la evaluación del efecto que tiene sobre las características nutricionales, al someter a los constituyentes de interés de la semilla a un proceso industrial (p.ej. efectos térmicos sobre la calidad proteica); iii) la evaluación de los índices de digestibilidad y de energía metabolizable en diferentes etapas productivas de los animales; iv) la evaluación de las interacciones, así como concentraciones máximas permitidas, de compuestos anti nutricionales, evaluando sus efectos en los procesos digestivos y la fisiología animal; v) el análisis de su aporte nutricional al emplear la semilla o algún derivado nutricional obtenida de ella, como suplemento en dietas establecidas; vi) la evaluación de las propiedades nutricias de la semilla en un abanico más amplio de especies y razas de animales rumiantes y no rumiantes de interés pecuario (haciendo énfasis en un enfoque regional). 


\section{Agradecimientos}

Al Consejo Nacional de Ciencia y Tecnología (CONACYT) del gobierno de los Estados Unidos Mexicanos, por las facilidades técnicas y materiales derivadas del proyecto cátedras para jóvenes doctores (cátedra 2515).

\section{- Literatura citada:}

1. ONU-FAO. Organización de las Naciones Unidas para la Agricultura y la Alimentación. Agroindustrias para el desarrollo. $1^{\text {a }}$ ed. Roma, Italia: 2013.

2. García-Contreras AC, de Loera YG, Guevara JA, García C. Alimentación práctica del cerdo. Rev Complut Cienc Vet 2012;6(1):21-50.

3. Mosenthin R, Zentek J, Zebrowska T. Biology of nutrition in growing animals. In: Mosenthin R, et al. editors. Biology of growing animals. Series. $1^{\text {st }}$ ed. New York, USA: Elsevier; 2006:1-640.

4. Buitrago AJ. La yuca en la alimentación animal. $1^{\text {a }}$ ed. Cali, Santiago de Cali, Colombia: Centro Internacional de agricultura tropical (CIAT); 1990.

5. Borja E, Medel P. En: Rebollar PG, de Blas C, Mateos GG. editores. Avances en la alimentación del porcino. Curso de Especialización. Barcelona, España: Fundación Española para el Desarrollo de la Nutrición Animal (FEDNA); 1998:261-312.

6. Anaya LA. Ecología química. 1 ${ }^{\text {a }}$ ed. Ciudad de México, México: Plaza y Valdés; 2003.

7. Vadivel VG, Janardhanan K. Nutritional and antinutritional characteristics of seven south indian wild legumes. Plant Food Hum Nutr 2005;2(60):69-75.

8. Ranalli P. Improvement strategies of leguminosae biotechnology. $1^{\mathrm{a}}$ ed. New York, USA: Springer; 2003.

9. Liener IE. Implications of antinutritional components in soybeans foods. Crit Rev Food Sci Nutr 1994;34(1):31-67.

10. Huisman J, Tolman G. In: Garnsworthy P. et al editors. Antinutritional factors in the plant proteins of diets for non-ruminants. Recent advances in animal nutrition. $1^{\text {st }}$ ed. London, United Kingdom: Butterworth Heinemann; 1992:3-31. 
11. Castillo-Caamal J, Jiménez-Osornio J, López-Pérez A, Aguilar-Cordero W, CastilloCaamal A. Feeding Mucuna beans to small ruminants of mayan farmers in the Yucatán peninsula, México. Trop Subtrop Agroecosyst 2003;2(1):113-117.

12. Muinga RW, Saha HM, Mureithi JG. The effect of Mucuna (Mucuna pruriens) forage on the performance of lactating cows. Trop Subtrop Agroecosyst 2003;3(1):87-91.

13. Loyra-Tzab E. Sarmiento-Franco L, Sandoval-Castro C, Santos-Ricalde R. Nutrient digestibility and metabolizable energy content of Mucuna pruriens whole pods fed to growing pelibuey lambs. Asian Australas J Anim Sci 2013;26(7):981-986.

14. Ruiz-Sesma B, Belmar-Casso F, Mendoza-Nazar P, Rivera-Lorca J, Trejo-Lizama W, Herrera-Haro J, et al. Evaluación del frijol terciopelo (Stizolobuim deeringianum) remojado, en dietas para cerdos en crecimiento. Universidad y Ciencia 2009;2(25):141150.

15. Siddhuraju P, Becker K, Makkar HP. Studies on the nutritional composition and antinutritional factors of three different seed material of an under-utilised tropical legume, Mucuna pruriens var. utilis. J Agr Food Chem 2000;48(1):6048-6060.

16. Cannas A, Tedeschi L, Atzori A. In: Sauvant D, et al editors. The development and evaluation of the small ruminant nutrition system. Modelling nutrient digestion and utilization in farm animals. $1^{\text {st }}$ ed. Wageningen, the Netherlands: Academic Publishers; 2010:263-272.

17. Ferrell CL, Jenkins TG. Body composition and energy utilization by steers of diverse genotypes fed a high-concentrate diet during the finishing period: I. Angus, Belgian Blue, Hereford, and Piedmontese sires. J Anim Sci 1998;76(1):637-646.

18. Duarte VF. Estimación de los requerimientos energéticos del borrego Pelibuey en crecimiento para la adecuación del modelo CNCPS/SRNS [tesis doctoral]. Mérida, Yucatán: Universidad Autónoma de Yucatán; 2007.

19. Ezeagu IE, Maziya-Dixon B, Tarawali G. Seed characteristics and nutrient and antinutrient composition of 12 Mucuna accessories from Nigeria. Trop Subtrop Agroecosyst 2003;23(1):129-139.

20. Adebowale YA, Adeyemi A, Oshodi AA. Variability in the physicochemical, nutritional and anti nutritional attributes of six mucuna species. Food Chem 2005;89(1):37-48.

21. Latham MC. Nutrición humana en el mundo en desarrollo. Colección ONUFAO, alimentación y nutrición No. 29. $1^{a}$ ed. Roma, Roma, Italia: Organización de las Naciones Unidas para la Agricultura y la Alimentación; 2002. 
22. Calsamiglia S, Ferret A, Bach A. Tablas FEDNA de valor nutritivo de forrajes y subproductos fibrosos húmedos. Madrid, España; 2004.

23. Guzmán AH, Acosta JA, Alvarez-Muñoz Ma de A, García-Delgado S, Loarca-Piña G. Calidad alimentaria y potencial nutracéutico del frijol común. Agric Tec Mex 2002;28(2):159-173.

24. Vargas-Torres A, Osorio-Díaz P, Agama-Acevedo E, Morales-Franco L, Bello-Pérez L. Digestibilidad del almidón en diferentes variedades de frijol (Phaseolus vulgaris L.). Interciencia 2006;31(12):881-884.

25. Bondi AA. Nutrición animal. 1ª ed. Zaragoza, España: Editorial Acribia; 1989.

26. Caravaca R. Catel G. Guzmán G. Delgado P. Mena G. Alcalde A. González R. Bases para la producción animal, serie de manuales universitarios número 61 . $1^{\mathrm{a}}$ reimpresión. Sevilla, España: Universidad de Sevilla; 2005.

27. Agudelo GG. Fundamentos de nutrición animal aplicada. $1^{\mathrm{a}}$ ed. Antioquia, Colombia: Universidad de Antioquia Editorial Universidad; 2001.

28. Van Lier E, Regueiro M. Digestión en el retículo-rumen. Departamento de producción animal y pasturas. Series de anatomía y fisiología animal. 1. ed. Facultad de Agronomía. Montevideo, Rio de la Plata, Uruguay: Universidad de la República editores. 2008;130.

29. Van Lunan T, Cole D. Energy-amino acid interactions in modern pig genotypes. In: Garnsworthy $\mathrm{P}$, et al. editors. Recent advances in animal nutrition. $1^{\text {st }}$ ed. Nottingham, United Kingdom: Nottingham University Press; 1996:233-261.

30. Chaparro AS, Aristizábal TI, Gil GJ. Composición y factores antinutricionales de las semillas del género mucuna. Rev Fac Nac Agron 2009;62(1):4843-4853.

31. Siddhuraju $P$, Becker K. Nutritional and antinutritional composition, in vitro amino acid availability, starch digestibility and predicted glycemic index of differentially processed mucuna beans (Mucuna pruriens var. Utilis): an under-utilised legume. Food Chem 2005;91(2):275-286.

32. Vadivel V, Janardhanan K. Nutritional and anti-nutritional composition of velvet bean: an under-utilized food legume in south India. Int J Food Sci Nutr 2005;4(1):279-87.

33. Janardhanan K, Gurumoorthi P, Pugalenthi M. Nutritional potential of five accessions of a South Indian tribal pulse, Mucuna pruriens var. Utilis. The effect of processing methods on the content of L-Dopa, phytic acid and oligosaccharides. Trop Subtrop Agroecosyst 2003;(1):141-152. 
34. Church DC. Pond WG. Pond KR. Fundamentos de nutrición y alimentación de animales. $2^{\mathrm{a}}$ ed. México, DF: Limusa; 1995.

35. Mohan VR, Janardhanan K. Chemical analysis and nutritional assessment of lesser known pulses of the genus Mucuna. Food Chem 1995;52(1):275-280.

36. Ezeagu IE, Maziya-Dixon B, Tarawali G. Seed characteristics and nutrient and antinutrient composition of 12 Mucuna accessories from Nigeria. Trop Subtrop Agroecosyst 2003;23(1):129-139.

37. Tacon GJ. Nutrición y alimentación de peces y camarones cultivados. En: Organización de la Naciones Unidas para la Agricultura y la Alimentación (ONUFAO). Manual de capacitación. Proyecto GCP/RLA/102/ITA apoyo a las actividades regionales de acuicultura para América Latina y El Caribe. $1^{a}$ ed. Brasilia, Brasil: 1989;189-210.

38. Felton EE, Kerley MS. Performance and carcass quality of steers fed different sources of dietary fat. J Anim Sci 2004;82(6):1794-1805.

39. Raes KL, Haak A. Balcaen E, Claeys D, Demeyer S. Effect of feeding linseed at similar linoleic acid levels on the fatty acid composition of double-muscled Belgian Blue young bulls. Meat Sci 2004;66(1):307-315.

40. Palmquist D. Use of fats in diets for lactating dairy cows. In: Wiseman J. Fats in animal nutrition. $1^{\text {st }}$ ed. London, United Kingdom: Butterworths; 1984.

41. Lorenzetti F, MacIsaac S, Arnason JT, Awang DVC, Buckles D. In: Buckles D, Eteka A, Osiname O, Galiba M, Galiano N. The phytochemistry, toxicology and food potential of velvetbean (Mucuna spp., Fabaceae). Cove Crops in West Africa: contributing to sustainable agriculture. International Development Research Center (IDRC), International Institute of Tropical Agriucltura (IITA) y Sasakawa Global; 1998;67-84.

42. Wichers HJ, Wijnsma R, Visser JF, Malingre THM, Huizing HJ. Production of LDOPA by cell suspension cultures of Mucuna pruriens. Effect of environmental parameters on the production of L-DOPA. Plant Cell Tiss Org 1985;4(1):75-82.

43. Mugendi JB, Njagi EN, Kuria EN, Mwasaru MA, Mureithi JG, Apostolides Z. Effects of processing technique on the nutritional composition and anti-nutrient content of mucuna bean (Mucuna pruriens L.). Afr J Food Sci 2010;4(4):156-166.

44. Suárez MM, Kizlansky A, López LB. Evaluación de la calidad de las proteínas en los alimentos calculando el escore de aminoácidos corregido por digestibilidad. Nutr Hosp 2006;21(1):47-51.

45. Trejo LW. Strategies to improve the use of limited nutrient resources in pig production in the tropics. J Agr Rural Dev Trop 2005;(Suppl 85):49-68. 
46. Encalada MP. Uso de frijol terciopelo (Mucuna pruriens) tostado y suplementado con vitamina B6 en raciones para pollos de engorde [tesis licenciatura]. San Antonio de Oriente, Honduras: Universidad Zamorano, Escuela Agrícola Panamericana Zamorano; 2002.

47. García LR, Roque RG, González RM. Forraje de mucuna (Stysolobium aterrimun) para la alimentación de vacas Holstein. Rev Cub Cien Agr 2002;36(1):37-40.

48. Liener IE. Implications of antinutritional components in soybeans foods. Crit Rev Food Sci Nutr 1994;34(1):31-67.

49. Jiménez-Escring A, Jiménez-Jiménez I, Pulido R, Saura-Calixto F. Antioxidant activity of fresh and processed edible seaweeds. J Sci Food Agric 2001;81(11):530-534.

50. Lohrmann NL, Logan BA, Johnson As. Seasonal acclimatization of antioxidants and photosynthesis in Chondrus crispus and Mastocarpus stellatus, two co-occurring red algae with differing stress tolerances. Biol Bull 2004;207(1):225-232.

51. Farougou S, Kpodekon M, Tokannou R, Djossou VD, Akoutey A, Youssao IA. Utilisation de la farine de Mucuna pruriens (L.) DC dans l'aliment de croissance des pintades (Numida meleagris). Rev Méd Vét; 2006:157(1):502-508.

52. Del Carmen J, Gernat AG, Myhrman R, Carew LB. Evaluation of raw and heated Velvet Beans (Mucuna pruriens) as feed ingredients for broilers. Poultry Sci 1999;78(1):866872.

53. Emenalom OO, Udedabie AB, Esonu BO, Etuk EB, Emenike HI. Evaluation of unprocessed and cracked, soaked and cooked velvet beans (Mucuna pruriens) as feed ingredients for pigs. Livestock Res Rural Dev 2004;16(5):33-39.

54. Grant G, Dorward PM, Buchan WC, Amour JC, Pusztai A. Consumption of diets containing raw soya beans (Glycine max), kidney beans (Phaseolus vulgaris), cowpeas (Vigna unguiculata) or lupin seeds (Lupinus angustifolius) by rats for up to 700 days: effects on body composition and organ weights. Brit J Nutr 1995;73(1):17-29.

55. Lohrmann NL, Logan BA, Johnson As. Seasonal acclimatization of antioxidants and photosynthesis in Chondrus crispus and Mastocarpus stellatus, two co-occurring red algae with differing stress tolerances. Biol Bull 2004;207(1):225-232.

56. Mary-Josephine R, Janardhanan K. Studies on chemical composition and antinutritional factors in three germplasm seed materials of the tribal pulse, Mucuna pruriens (L.) DC. Food Chem 1992;43(1):13-18. 